\title{
Improved Halftone Image Data Hiding with Intensity Selection
}

\author{
Ming Sun Fu, Oscar C. Au \\ Department of Electrical and Electronic Engineering, \\ Hong Kong University of Science and Technology, \\ Clear Water Bay, Hong Kong, China. \\ Tel: +852 2358-7053,Fax: +852 2358-1485,Email:fmsun@ust.hk, ecau@ust.hk
}

\begin{abstract}
:
In this paper, we propose a novel algorithm called intensity selection (IS) that can be applied to three existing halftone image data hiding algorithms DHST, DHPT and DHSPT to achieve improved visual quality. The proposed IS algorithm generalizes the hidden data representation and select the best location out of a set of candidate locations for the application of DHST/DHPT/DHSPT. It chooses pixel locations that are either very bright or very dark. The IS requires the inverse-halftoned image which implies potentially high computation requirement. Experiments suggest that significant improvement in visual quality can be achieved.
\end{abstract}

\section{Introduction}

Nowadays, images appear routinely in massively distributed printed matters such as books, magazines, newspapers, printer outputs and fax documents. Images appear also in widely accessible web pages, and multimedia files on the Internet and in storage media such as CD-ROM and DVD. Associated with the widespread circulation of images are issues of copyright infringement, authentication and privacy. One of the possible solutions is to embed some hidden watermarking data into the images.

Digital watermarking [1] is a process that can embed some invisible digital data called watermark into an image. In this paper, we are concerned about invisible watermarking for a special kind of image: halftone images. Halftoning [2] is a process to change multi-tone images into 2 tone images, which look like the original multi-tone images when viewed from a distance. Halftone images are widely used in the printing of books, magazines, newspapers and in computer printers, which are very common in our daily lives. It is often desirable to hide certain invisible meta-data within the halftone images such as company identity, owner information, creation date and time and other information for copyright protection and authentication purposes. As most halftone images are printed on papers, there are very few, if any, possible digital attacks on the watermarked halftone images though there can be many physical attacks on the papers on which the images are printed. As a result, robustness or fragileness of halftone image watermarking schemes is not particularly important for many applications. Instead, the data hiding capacity and image quality are much more important in most applications. In this paper, we assume that there are no physical attacks and the watermarked halftone images can be fully recovered without any error by scanning the printed images.
There are two main kinds of halftoning techniques, namely, ordered dithering [3] and error diffusion [14]. It is well known that most image processing techniques such as filtering or resizing cannot be applied to a halftone image to produce another halftone image with good visual quality. Watermarking is not an exception. Most, if not all, existing watermarking schemes for natural images as mentioned earlier cannot be applied to halftone images due to their special characteristics. The halftone image pixels take on only two values, typically 0 (black) and 255 (white), resulting in lots of high frequency noise and little intensity redundancy. Most existing watermarking schemes for natural images would generate multi-tone, instead of halftone, images.

However, there are still some existing techniques for halftone image watermarking. Some used two different dithering matrices for the halftone generation [3] such that the different statistical properties due to the two matrices can be detected. Some used stochastic screen patterns [4] and conjugate screen patterns [5] in which two screens were used to form two halftone images and the data was embedded through the correlations between two screens. We also proposed some methods for hiding a fairly large amount of data in pseudo-random locations in halftone images without knowledge of the original multi-tone image and the halftoning method. They were called DHST[6], DHPT[6], DHSPT[7]. While DHST suffered from severe "salt-andpepper' artifacts, DHPT is better and DHSPT is even better with few remaining "salt-and-pepper" artifacts.

In this paper, we propose a method called Intensity Selection (IS) that can be applied to DHST, DHPT and DHSPT to achieve improved visual quality. In Section 2, the DHST, DHPT and DHSPT will be reviewed. The proposed method, IS, will be introduced. In section 3, the simulation results will be presented and discussed.

\section{Review of DHST, DHPT, DHSPT}

In DHST, DHPT and DHSPT, one halftone pixel is used to store 1 data bit (e.g. black=0, white $=1$ ). In DHST, N data bits are hidden at $\mathrm{N}$ pseudo-random locations by forced toggling. In other words, when the original halftone pixel at the pseudo-random location differs from the desired value, it is forced to toggle. This results in lots of clusters of pixels of the same color, some white and some black. These clusters are called the "salt-and-pepper" artifacts. They are visually disturbing to the human eye. To retrieve the data, the data is simply read from the $\mathrm{N}$ pseudo-random locations. 
DHPT improves over DHST by performing pair toggling instead of forced toggling at the pseudo-random locations. When toggling is need, DHPT toggles a complementary pair (one white and one black) instead of just one pixel. The complementary pixel is chosen randomly from the candidates within the $3 \times 3$ neighborhood. The complementary toggling preserves the local intensity and reduces the "salt-and-pepper" artifacts significantly.

DHSPT improves upon DHPT by choosing the complementary pixel in a 'smart' way so that the salt-andpepper clusters are smaller. A quantity called 'connection' is computed for each of complementary pixel candidates and the one with largest before-toggle 'connection' is chosen. Basically, if a pixel is maximally connected with neighboring pixels, it would be least connected after toggling and the resulting cluster would be of the smallest size. The visual quality of DHSPT is significantly better than that of DHPT, as shown in Fig. 3.

\section{Proposed Intensity Selection}

To improve the visual quality of DHST, DHPT and DHSPT, we propose to modify the data representation. We propose to hide one bit of the embedded data at $M$ pseudorandom locations instead of just one location. Instead of generating $N$ pseudo-random locations, we generate $N M$ pseudo-random locations which are divided into $N$ groups of $M$ locations. Then one bit is embedded in each group of $M$ locations by forcing the parity of the sum of the $M$ pixels to be even or odd according to the data bit to be embedded. The parity of the $M$-pixel-sum is changed, when needed, by two steps. The first step is Intensity Selection (IS) that selects one out of the $M$ pixels to be changed, basing the decision on the intensity condition of the original multi-tone image. The second step is the toggling of the selected pixel using DHST, DHPT or DHSPT. This corresponding algorithms are called DHST-IS, DHPT-IS and DHSPT-IS.

Assume that the embedded data bits and the original halftone image pixels are statistically independent and that each embedded data bit is equally likely to be 0 or 1 . Then, with a probability of 0.5 , the original $M$-pixel-sum parity is the desired value and thus no change is needed. With a probability of 0.5 , the original $M$-pixel-sum parity is different from the desired value. In this case, the proposed IS and the toggling are applied to force the $M$-pixel-sum parity to be the desired value. To read the embedded data, one simply uses the same random number generator and the same seed to obtain the $N M$ pseudo-random locations. Then the embedded data bits can be extracted by examining the $M$-pixel-sum parity.

Let $\left\{\left(i_{1}, j_{1}\right),\left(i_{2}, j_{2}\right), \ldots,\left(i_{M}, j_{M}\right)\right\}$ be $M$ pseudorandom locations among which one data bit needs to be hidden. These locations are typically far apart, locating at different parts of the image. Let $x(i, j), y(i, j)$ be the original multi-tone image pixel value, and the halftone value at location $(i, j)$ respectively. The $M$-pixel-sum parity of

$$
\begin{gathered}
\left\{y\left(i_{1}, j_{1}\right), y\left(i_{2}, j_{2}\right), \ldots, y\left(i_{M}, j_{M}\right)\right\} \text { is } \\
\left(\sum_{k=1}^{M} y\left(i_{k}, j_{k}\right)\right) \bmod 2
\end{gathered}
$$

When the parity is different from the desired value, an odd number of the halftone values $y(i, j)$ should be toggled to change the parity. To minimize the distortion due to the changes, only one of the $M$ pixels will be changed. We will call this pixel location $(m, n)$. With $M$ locations available, the proposed IS seeks to choose the location at which the distortion due to DHST/DHPT/DHSPT would be least visually annoying.

To motivate IS, we will analyze the distortion patterns of DHSPT, DHPT and DHST first. Often within a small neighborhood, the original multi-tone image pixels are approximately constant. Suppose $x(m+i, n+j)=K$ for some $0 \leq K \leq 255$ and $(i, j) \in \Psi$ where $\Psi$ is a local neighborhood of $(m, n)$. Then any good halftone method would have approximately a fraction $K / 255$ of the pixels within $\Psi$ being white and $(1-K) / 255$ being black so that the local average intensity is approximately $K$.

Consider the mid-gray regions with $K$ close to 127 . There are typically many black pixels and white pixels both of which somewhat evenly distributed, forming some regular structure or texture. When DHST is applied, it may force a black pixel to be white or a white pixel to be black depending on the data bit to be embedded. Regardless of the color, the result is that the regular structure is locally punctured resulting usually in a visually disturbing large cluster. The cluster size can range from 2 to more than 10 . The clusters in real images often contain 3, 4 or 5 pixels which are large enough to be visually disturbing, especially against a regular background. And since the background is mid-gray, both black and white clusters are visually disturbing. The clusters tend to be smaller on the average for DHPT and even smaller for DHSPT. Still the punctured regular structure can be very annoying.

While DHST/DHPT/DHSPT introduces distortions inevitably whenever toggling is applied, the resulting 'saltand-pepper' clusters tend to be larger and visually more disturbing in mid-gray area than in bright or dark areas. Midgray area tend to contain highly regular patterns such as checker-board patterns in which even a tiny cluster can be very annoying. The dark regions on the other hand tend to contain a lot of black pixels and thus small changes are much less visible. And the same for bright regions. As a result, our proposed Intensity Selection (IS) tries to select locations that are either very bright or very dark for best visual quality.

Suppose that the original multi-tone pixels $\left\{x\left(i_{1}, j_{1}\right), x\left(i_{2}, j_{2}\right), \ldots, x\left(i_{M}, j_{M}\right)\right\}$ are available. We define the eccentricity at location $(m, n)$ as

$$
\text { eccentricity }(m, n)=|x(m, n)-127|
$$


When eccentricity is small, the pixel is mid-gray and close to 127. When eccentricity is large, the pixel is either very bright or very dark. In IS, we choose the location with the largest eccentricity among the $\mathrm{M}$ candidates.

But DHST, DHPT and DHSPT are designed for cases in which the original halftone images are not available. In those cases, the proposed IS cannot be applied due to the absence of the original image. Here we also propose a modified version of IS which does not need the original images.

The modification is to perform inverse halftoning [8] on the halftone image to obtain an estimate of the original multi-tone image, and then perform IS based on the estimated multi-tone image. While inverse halftoned images are not perfect, our experiments suggest that they are good enough for the proposed IS. The eccentricity tends to be insensitive to inverse halftoning distortion. One price of the modification is the large computational requirement of inverse halftoning.

While there are many existing inverse-halftoning methods such as projection onto convex set (POCS), wavelet-based methods and edge-preserving filtering[5], we find in our experiments that simple lowpass filtering is sufficient to give good enough estimated values for IS. And lowpass filtering has the lowest complexity among all inverse-halftoning methods. In addition, lowpass filtering need to be applied only at the pseudo-random locations. Thus the computation complexity can be further reduced. The algorithm that applies inverse halftoning, IS and DHST/DHPT/DHSPT are simply called DHST-IS, DHPT-IS and DHSPT-IS respectively.

\section{Simulation results}

Figure 1 is a portion of the $512 \times 512$ error diffused halftone 'Lena' with 4096 bits embedded by DHST. The kernel used is Steinberg. Many "salt-and-pepper" artifacts can be easily seen. In Figure 2, the proposed Intensity Selection with $M=4$ is applied to DHST to hide 4096 bits. The DHST-IS is based on the original image. The salt-andpepper artifacts are significantly reduced. This shows the effectiveness of the proposed IS.

In Figure 3, DHPT is applied to hide 4096 bits in the $512 \times 512$ Lena. Visual quality improvement over DHST can be observed. In Figure 4 , the proposed Intensity Selection with $M=4$ is applied to DHPT to hide 4096 bits. The DHPTIS is based on the original image. Again, the salt-and-pepper artifacts are significantly reduced.

In Figure 5, DHSPT is applied to hide 4096 bits in Lena. Visual quality improvement over DHPT can be observed again. The IS is based on the original image. Visual quality of DHSPT is reasonably good. However, as the proposed IS with $M=4$ is applied in Figure 6 , the visual quality is significantly improved. The salt-and-pepper artifacts are greatly reduced. With the proposed IS, DHPT-IS and DHSPT-IS have similar visual quality.

Our experiment results also show that IS based on the original multi-tone image has essentially the same visual quality as IS based on the inverse halftoned image. Due to limited space, the images are not shown.

Overall, the results suggest that the proposed Intensity Selection with $M=4$ is effectively in improving the visual quality of DHST, DHPT and DHSPT. In general, large M will give large improvement as there are more pixels available for choosing.

\section{Conclusion}

In this paper, we propose the Intensity Selection (IS) to improve the visual quality of DHST, DHPT and DHSPT. IS represents a data bit as the parity of the sum of the halftone pixels at $M$ pseudo-random locations and selects the best out of the $M$ possible locations to apply DHST/DHPT/DHSPT. In the experiments, IS is found to be very effective in improving the visual quality of DHST, DHPT and DHSPT without affecting the data hiding capacity. In general, the visual quality of IS is better for larger $M$.

\section{Acknowledgement}

This work is funded by an RGC CERG grant.

\section{References}

[1] F. Mintzer, et al., "Effective and Ineffective Digital Watermarks", Proc. of IEEE Int. Conf. on Image Processing, Vol. 3, Oct. 1997, pp. 9-13.

[2] R. A. Ulichney, Digital Halftoning, Cambridge, MA, MIT Press, 1987.

[3] Z. Baharav, D. Shaked, "Watermarking of Dither Halftoned Images", Proc. of SPIE Security and Watermarking of Multimedia Contents, Vol. 1, pp. 307313, Jan. 1999.

[4] K.T. Knox, "Digital Watermarking Using Stochastic Screen Patterns", United States Patent Number $5,734,752$.

[5] S. G. Wang, "Digital Watermarking Using Conjugate Halftone Screens", United States Patent Number $5,790,703$.

[6] M.S. Fu, O.C. Au, "Data Hiding for Halftone Images", Proc. of SPIE Conf. On Security and Watermarking of Multimedia Contents II, Vol. 3971, pp. 228-236, Jan. 2000.

[7] M.S. Fu, O.C. Au, "Data Hiding by Smart Pair Toggling for Halftone Images", Proc. of IEEE Int. Conf. On Acoustics, Speech and Signal Processing, Vol. 4, pp. 2318-2321, 5-9 Jun. 2000.

[8] O.C. Au, M.S. Fu, et. al., "Hybrid Inverse Halftoning using Adaptive Filtering", Proc. IEEE Int. Sym. On Circuits and Systems, Vol. 4, pp. 259-262, Jun. 1999. 

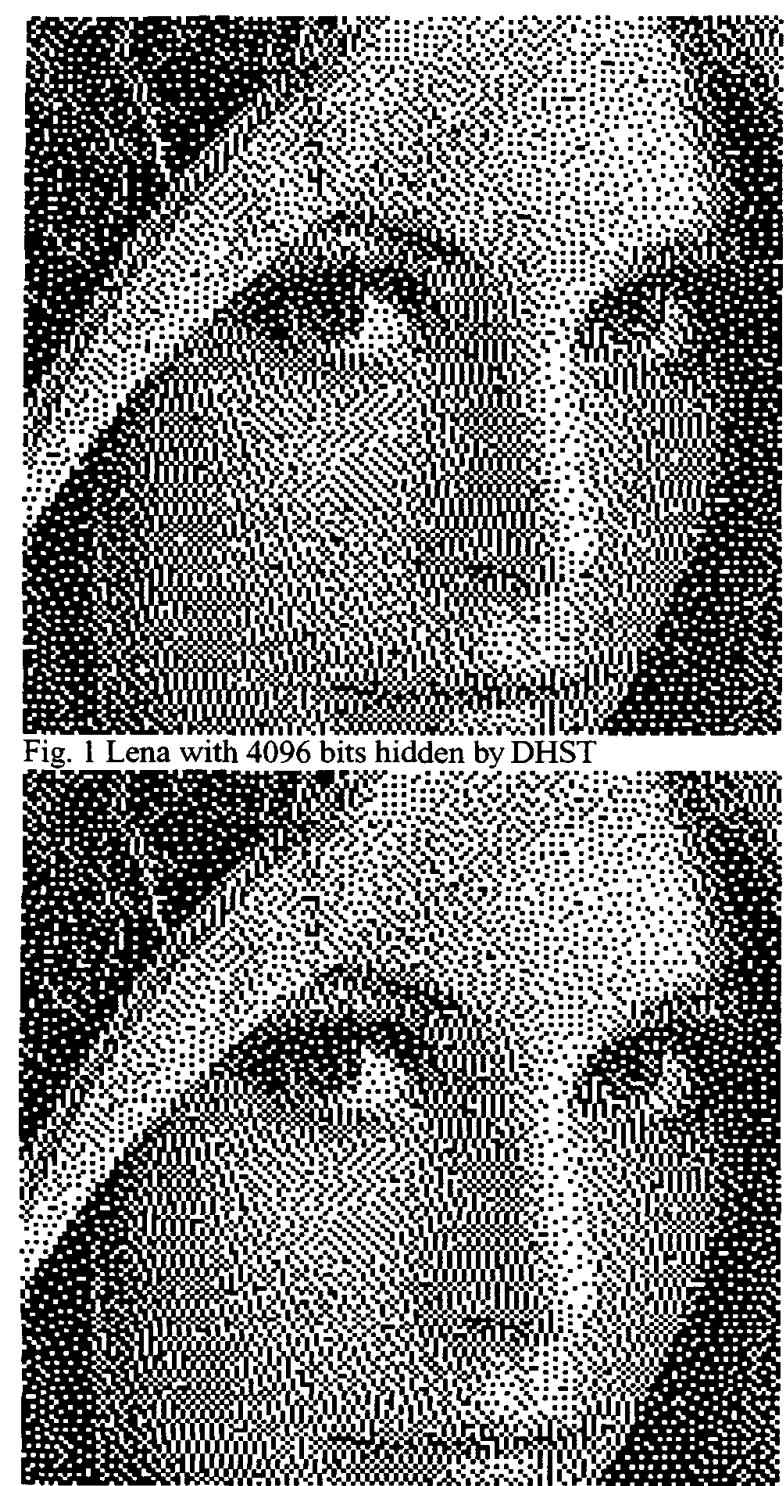

Fig. 3 Lena with 4096 bits hidden by DHPT

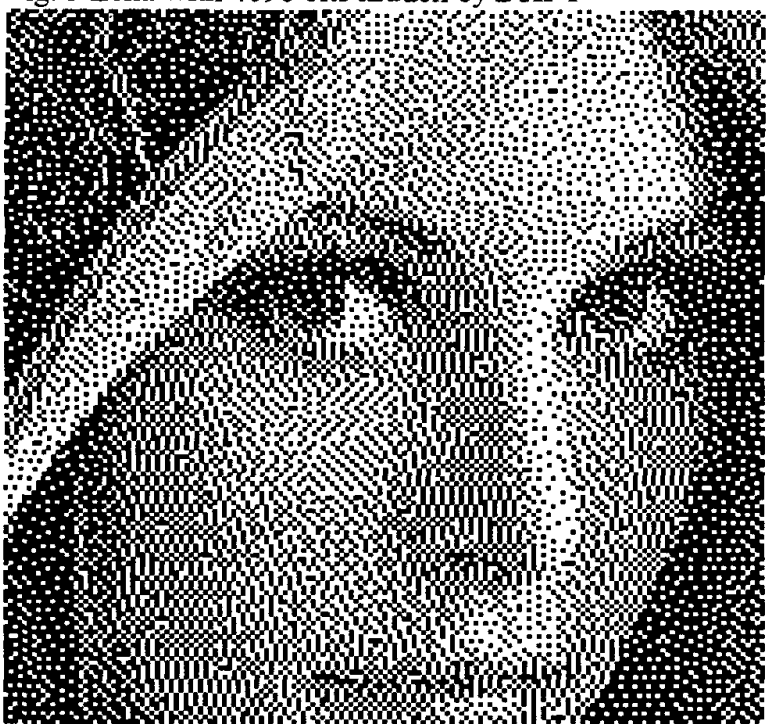

Fig. 5 Lena with 4096 bits hidden by DHSPT

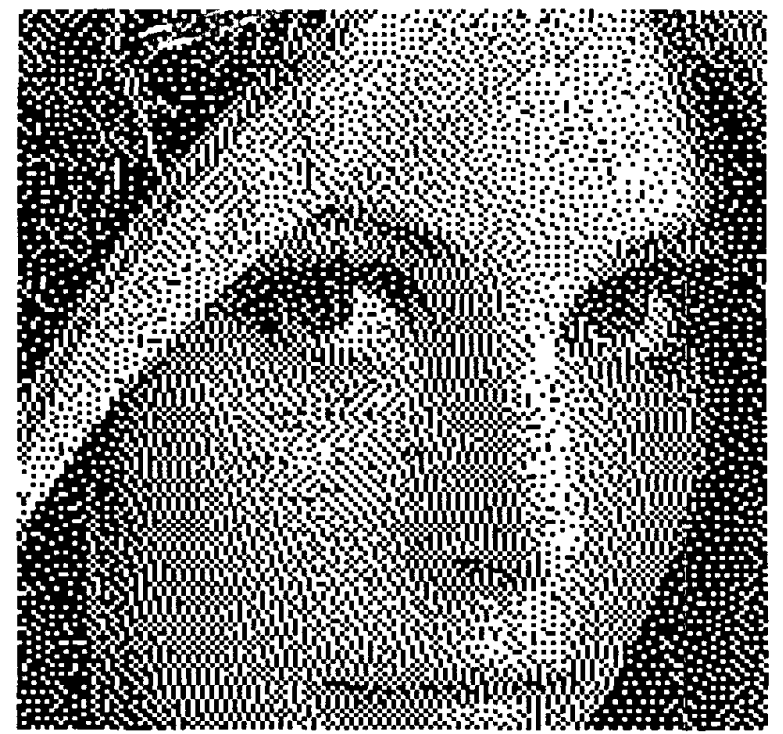

Fig. 2 Lena with 4096 bits hidden by DHST-IS $(M=4)$

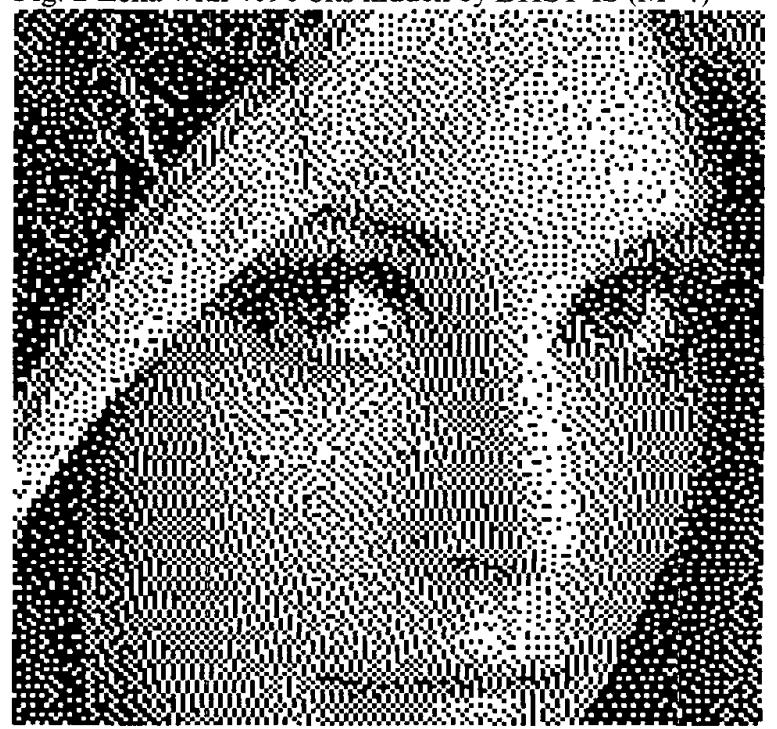

Fig. 4 Lena with 4096 bits hidden by DHPT-IS $(M=4)$

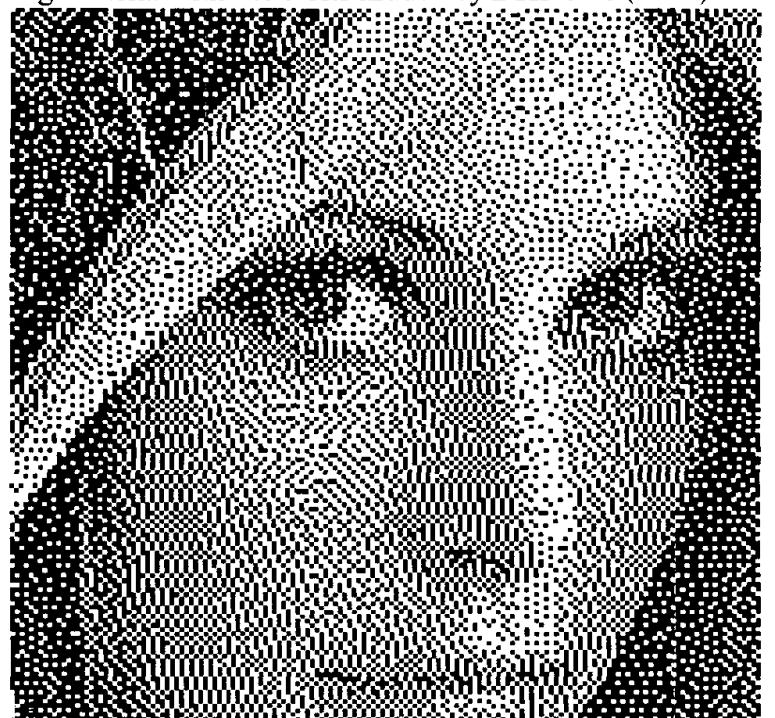

Fig. 6 Lena with 4096 bits hidden by DHSPT-IS $(M=4)$ 